\title{
Local thermodynamics of alcohols from THz-calorimetry: Spectroscopic fingerprints of entropic loss and enthalpic gain
}

\author{
Simone Pezzotti, ${ }^{1}$ Federico Sebastiani, ${ }^{1,2}$ Eliane P. van Dam, ${ }^{1}$ Sashary Ramos, ${ }^{1}$ Valeria Conti Nibali, ${ }^{1,3}$ Gerhard \\ Schwaab, ${ }^{1}$ and Martina Havenith ${ }^{1,}$ a) \\ 1) Department of Physical Chemistry II, Ruhr University Bochum, Bochum, Germany \\ ${ }^{2)}$ Current affiliation: Department of Chemistry "U. Schiff”, University of Florence, I-50019 Sesto Fiorentino (FI), \\ Italy \\ ${ }^{3)}$ Current affiliation: Dipartimento di Scienze Matematiche e Informatiche, Scienze \\ Fisiche e Scienze della Terra (MIFT), Università di Messina, 98166 Messina, \\ Italy
}

Hydration free energies are dictated by a subtle balance of hydrophobic and hydrophilic interactions. which is crucial for many biological processes and technological applications, such as protein folding and molecular recognition. Whereas so far the overall entropy and enthalpy are experimentally determined based on equilibrium measurements using a calorimeter, we present here a pure spectroscopic access to these important observables, which give direct access to the underlying molecular mechanism that determines these driving forces. Using $\mathrm{THz}$ calorimetry the contributions due to cavity formation and hydrophilic interactions can be traced back to changes in the intermolecular hydrogen bond stretching region around $150-200 \mathrm{~cm}^{-1}$ and spectroscopic changes due to strong solute-water interactions in the frequency range of the librational modes, i.e. between 540 and $600 \mathrm{~cm}^{-1}$. Thus, we are able to link the thermodynamic model of the Lum-Chandler-Weeks theory, which was a pure "Gedankenexperiment", directly to experimental observables. We show that alcohol hydration can be described by a sum of a free energy cost of forming and wrapping a cavity around the solute (which is entropic for small alcohols) and an enthalpic gain due to the hydrogen bonds formed between the alcohol $\mathrm{OH}$ group and bound water molecules around it. In the future, our approach will allow to quantify entropic cost and enthalpic gain not only in equilibrium but also in non-equilibrium processes.

Hydrophobic hydration is important to understand and predict fundamental biological processes, such as protein folding and aggregation ${ }^{1-3}$, molecular recognition $^{4-6}$ and liquid-liquid phase separation, ${ }^{7-9}$ as well as in many other fields, e.g. water-mediated catalysis ${ }^{10-12}$ and electro-catalysis ${ }^{13-15}$. Going from small solutes all the way up to large biomolecules, a subtle balance between hydrophilic and hydrophobic interactions is what dictates hydration free energies. ${ }^{16-24}$ Evaluating such balance requires a local mapping of hydrophilic and hydrophobic contributions, which remains a challenge for both theory and experiments. ${ }^{18,19,25-29}$ From the experimental side, these contributions are notoriously difficult to probe and cannot be dissected by standard calorimetry approaches. Nevertheless, several attempts have been undertaken since the ability to characterize hydration thermodynamic properties would guarantee major economic advantages for e.g. drug discovery. ${ }^{30}$ In the present work, we seek for a pathway to experimentally access both hydrophilic and hydrophobic contributions via $\mathrm{THz}$-calorimentry ${ }^{31}$ (i.e. via correlating $\mathrm{THz}$ vibrational fingerprints with entropy and enthalpy) and evaluate their synergy in dictating hydration free energies within a simple thermodynamic model.

A step-wise way to separate hydrophobic and hydrophilic contributions is to look at the hydration of a

\footnotetext{
a)Electronic mail: martina.havenith@rub.de
}

generic solute by adopting a thermodynamic cycle as illustrated in fig. $1 .^{32}$ It consists of a two-step process where (1) the water hydrogen bond (HB)-network is perturbed in order to create a cavity that can accommodate the solute; (2) the solute is inserted at the centre of the cavity, switching on attractive solute-water interactions. The total hydration energy is then given as the sum of the free energy of these two steps:

$$
\Delta \mu_{\text {hyd }}=\Delta \mu_{\text {cavity }}+\Delta \mu_{\text {bound }}
$$

The key aspect of this cycle is that $\Delta \mu_{\text {bound }}$ associated with step (2) is zero for a purely hydrophobic solute, which means that hydrophobic hydration is fully described by step (1), only. This latter step, which is associated to the free energy cost of cavity formation $\Delta \mu_{\text {cavity }}$, has been rationalized by the Lum-ChandlerWeeks theory: ${ }^{33}$ the hydration of small solutes forming cavities of $<1 \mathrm{~nm}$ radius requires a distortion of the water HB-Network, with associated entropic cost that scales with the cavity volume, while the solvation of larger solutes induces formation of water dangling $\mathrm{OH}$-groups, at an enthalpic cost that scales with the cavity surface area.

Building on our previous works, ${ }^{27,31}$ we aim for a direct experimental characterization of the hydrophilic and hydrophobic contributions described by each of the two steps of fig.1 and their interplay to determine the hydration free energy of molecules composed of both polar and apolar groups. Our study focuses on aqueous alcohol solutions as model systems, but the developed methodology can be generalized to more complex molecules and bio-interfaces, where the balance 
and distribution of polar and apolar groups are crucial parameters in determining hydration free energies and biological function. ${ }^{16,18,26}$
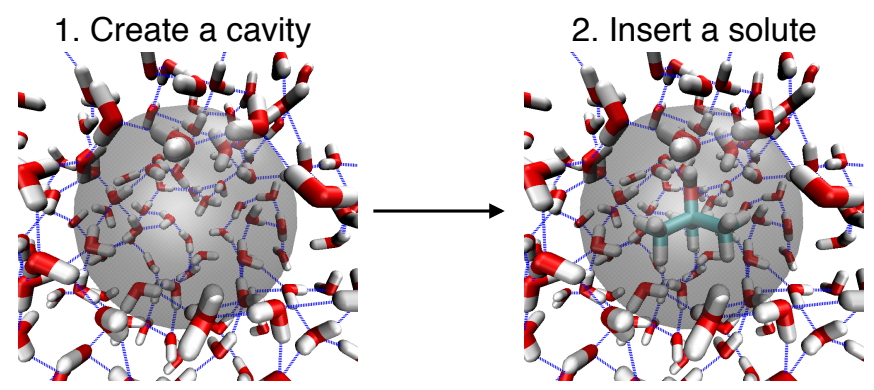

FIG. 1. The hydration free energy of a generic solute can be modelled as a two-step process: (1) create a cavity in water where the solute can be accommodated, with associated free energy cost $\Delta \mu_{\text {cavity; }}$ (2) insert a solute (tBuOH in the example) at the center of the cavity, with associated free energy gain $\Delta \mu_{\text {bound. }}$ Hydration water molecules are illustrated with red oxygen and white hydrogen, H-Bonds are in blue. The alcohol $(\mathrm{tBuOH})$ solute in step 2 is depicted with red oxygen, white hydrogen and cyan carbon atoms.

We have previously introduced $\mathrm{THz}$ absorption spectroscopy to characterize the water HB-network formed around hydrated solutes. ${ }^{31,34}$ The $\mathrm{THz}$ spectrum of the solute and its hydration shell is obtained by subtracting the volume-scaled absorption spectrum of bulk water from the absorption spectrum of a solution containing the solute of interest. ${ }^{27,31}$ Hydration-shell vibrational spectroscopies provide valuable information on the intermolecular interactions involving the solute and its hydration water molecules. ${ }^{31,35,36}$ In our previous studies we focused on the HB intermolecular stretching region (100-300 $\left.\mathrm{cm}^{-1}\right)$, i.e. the low frequency region showing the collective vibrations of the water HB-network, and we were able to identify two characteristic bands of the HB-network in the alcohol hydration layer. These were associated to $\mathrm{HBs}$ that spectroscopically, structurally and dynamically differ from the HBs formed in bulk liquid water. We observed these two characteristic bands for all alcohols from Methanol to Pentanol at varying concentration and temperature ${ }^{27,31}$, and similarly for clathrate hydrates ${ }^{34}$ and for water interacting with extended hydrophobic surfaces. ${ }^{37}$

Specifically, water molecules in the closest proximity of the alcohol create a network of HBs wrapped around the whole solute (covering both hydrophilic $\mathrm{OH}$ and hydrophobic $\mathrm{CH}_{2} / \mathrm{CH}_{3}$ groups). This cavity forming HB-wrap provides a specific $\mathrm{THz}$ contribution centred at $\sim 164 \mathrm{~cm}^{-1}$. A second population of HBs (HB-2bulk) connects hydration water to the bulk, and provides a specific $\mathrm{THz}$ contribution centred at $\sim 195 \mathrm{~cm}^{-1}$, which is almost bulk like, with a narrower linewidth indicative of a reduced configurational space. ${ }^{11,27} \mathrm{By}$ THz-calorimetry we could quantitatively correlate the solvation entropy of all the alcohols to the amplitude of the two identified $\mathrm{THz}$ fingerprints. We found, in agreement with predictions from the LCW theory, that the entropic cost of alcohol hydration is dominated by the process of forming and wrapping a cavity.

While this provided an access to thermodynamic quantities from spectroscopy, we will show here that we are able to decompose hydration entropy, as obtained experimentally from $\mathrm{THz}$-calorimetry, into the individual contributions from the $\mathrm{CH}_{3}, \mathrm{CH}_{2}$ and $\mathrm{OH}$ groups of alcohol molecules. This approach is suitable to evaluate how much the strong attractive interactions formed between the alcohol $\mathrm{OH}$ group and water alter hydration entropy with respect to the limit of only hydrophobic groups being solvated. While the hydrogen bond stretch region probes the strength of $\mathrm{HBs}$, which is decreased for the HB-wrap, we show that the sterical restrictions for water molecules bound to the $\mathrm{OH}$ group (denoted hereafter bound waters) can be sensitively probed by the hindered reorientational motion of a single water molecule, i.e. the librational mode. Putting these results together makes it possible to experimentally map both steps of the thermodynamic cycle of fig.1, hence revealing the interplay between hydrophilic and hydrophobic solvation mechanisms.

Building-blocks THz-calorimetry approach. We consider alcohols to be composed of three building blocks: $\mathrm{CH}_{3}, \mathrm{CH}_{2}$ and $\mathrm{OH}$ groups (fig.2C). By using a large set of alcohols as database $(\mathrm{MeOH}$, EtOH, $\mathrm{PrOH}, \mathrm{BuOH}, \mathrm{tBuOH}, \mathrm{PeOH})$, we derive group-specific hydration entropies $\left(\Delta S_{h y d}\right)$ adopting a global fitting procedure detailed in section 4 of the SI. The idea is that $\Delta S_{h y d}$ of each alcohol can be rewritten as the sum of $\Delta S_{\text {hyd }}$ contributions from the building blocks composing it, i.e. $\Delta S_{h y d}=\sum \Delta S_{h y d}^{\text {group }}$. The approximation made here is that each group provides the same partial contribution $\left(\Delta S_{\text {hyd }}^{\text {group }}\right)$ for different alcohols. The hydration entropy values for all investigated alcohols have been previously obtained from $\mathrm{THz}$-calorimetry, ${ }^{27,31}$ and were found in excellent agreement with values from standard calorimetry. The working principles of $\mathrm{THz}$-calorimetry are described in details in the SI.

The obtained $\Delta S_{\text {hyd }}^{\text {group }}$ values are reported in Fig.2A. $\mathrm{CH}_{3}$ groups provide the largest contribution to the hydration entropy, followed by $\mathrm{OH}$, while $\mathrm{CH}_{2}$ groups contribute much less $\left(\sim 40 \%\right.$ of a $\left.\mathrm{CH}_{3}\right) . \Delta S_{\text {hyd }}^{\text {group }}$ values obtained from classical MD simulations by means of a $3 \mathrm{D}-2 \mathrm{PT}$ model ${ }^{27,38}$ (see SI, section 6 for details) are also reported for comparison (green curve). 3D-2PT allows to spatially resolve hydration entropy around hydrated alcohols. Group specific values are obtained by direct integration within the volume assigned to the hydration shell of each group. The validity of the building-blocks approximation used in the experimental procedure is testified by the good theory-experiments comparison. 
A
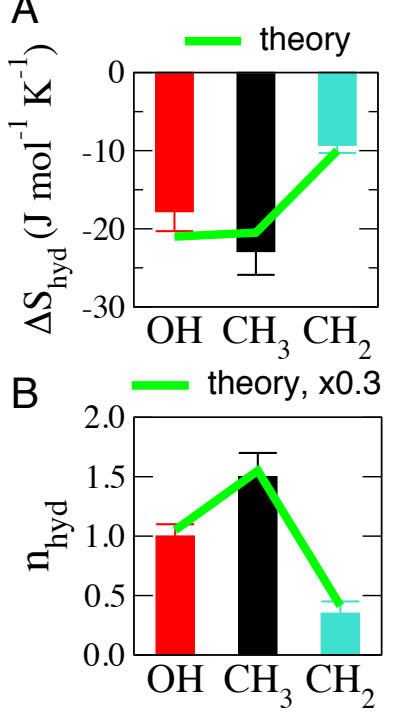

C
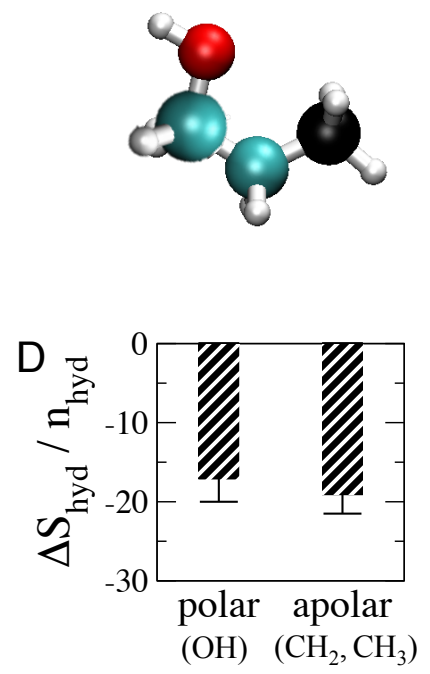

FIG. 2. Group specific entropy contributions (at room temperature) to alcohol hydration from THz-calorimetry. (A) individual contributions of $\mathrm{CH}_{3}, \mathrm{CH}_{2}$ and $\mathrm{OH}$ groups to hydration entropy, $\Delta S_{\text {hyd }}$. The green curve shows $\Delta S_{\text {hyd }}$ values predicted from classical MD simulations by means of a $3 D$ 2PT approach. (B) effective hydration numbers $\left(n_{\text {hyd }}\right)$ derived from $\mathrm{THz}$ experiments, compared to $n_{\text {hyd }}$ values from MD simulations (green). The theoretical $n_{\text {hyd }}$ values are arbitrarily scaled by 0.3 to compare with experiments, as they are obtained from direct counting of hydration water molecules while experimental values depend on spectroscopic activity. (C) illustration of the building blocks composing alcohols, i.e. $\mathrm{CH}_{3}$ (black), $\mathrm{OH}$ (red), $\mathrm{CH}_{2}$ (cyan). (D) Experimental $\Delta S_{\text {hyd }}$ values divided by $n_{\text {hyd }}$ and averaged separately over apolar $\left(\mathrm{CH}_{2}\right.$ and $\mathrm{CH}_{3}$ ) and polar (only $\mathrm{OH}$ ) groups.

The differences in hydration entropy for polar $(\mathrm{OH})$ and apolar $\left(\mathrm{CH}_{2}, \mathrm{CH}_{3}\right)$ groups are attributed to (i) a difference in the local water structure around the groups or (ii) a difference in the number of water molecules involved in the hydration of each group. Option (i) is unlikely to be the major contribution based on analysis of the structural, dynamical and vibrational properties of hydrated alcohols from ab initio and classical MD simulations. As shown in our previous work on hydrated tert-butanol, ${ }^{27}$ the water molecules around apolar $\left(\mathrm{CH}_{3}\right)$ and polar $(\mathrm{OH})$ groups all form on average 1.5 HB-wrap and 1.5 HB-2bulk. They further have homogeneous water-water HB-dynamics within the inner part of the hydration layer. However, bound water molecules interacting with the alcohol OH-group form an additional $\mathrm{HB}$ with the alcohol. In fig.2D, we show experimentallydeduced partial hydration numbers $\left(\mathrm{n}_{h y d}\right)$ for each group and we compare them with theoretical values obtained from direct counting of the water molecules in the hydration shell of each group (the values are averages over tBuOH, BuOH and $\mathrm{MeOH}$ ). The $\mathrm{CH}_{3}$ groups, which provide the largest contribution to hydration

entropy, also have the largest $\mathrm{n}_{h y d}$, and the trend of $\mathrm{n}_{h y d}$ mirrors that observed for $\Delta S_{h y d}$. If we divide $\Delta S_{h y d}$ by $\mathrm{n}_{\text {hyd }}$ and average over apolar $\left(\mathrm{CH}_{2}\right.$ and $\left.\mathrm{CH}_{3}\right)$ and polar (only $\mathrm{OH}$ ) groups separately, group-specific hydration entropy values per hydration water molecule for polar and apolar solvated moieties are obtained (fig.2D, which show no differences). Interestingly, Beyond the difference in the number of water molecules required to hydrate each group, we find that polar and apolar groups contribute equally to the hydration entropy of alcohols solutes. Thus, we propose that the entropic cost of alcohol hydration does not significantly depend on the specific $\mathrm{HB}$ interactions formed by the polar $\mathrm{OH}$ group with bound water molecules. The solute-bound water HBs, which are associated to step (2) of the thermodynamic cycle of fig.1, mostly contribute as an enthalpic gain to alcohol hydration, due to the additional HB that bound water molecules form with the alcohol $\mathrm{OH}$ functional group. This is confirmed by adopting our group-specific THz-calorimetry approach to decompose alcohol hydration enthalpy $\left(\Delta H_{h y d}\right)$ into $\mathrm{CH}_{2}, \mathrm{CH}_{3}$ and $\mathrm{OH}$ contributions (see SI, figure S2): we find that $\mathrm{OH}$ groups provide the dominant contribution $(-7.7 \pm 0.7$ $\mathrm{KJ} / \mathrm{mol}$ at room temperature) to $\Delta H_{h y d}$.

These results have important implications for the thermodynamic cycle of fig.1: the entropic cost of alcohol hydration is to a good approximation independent on step (2), i.e. independent on attractive alcohol-water interactions. In other words, the process of cavity formation, i.e step (1), dominates alcohol hydration entropy. This explains why LCW theory has been highly successful in predicting alcohol hydration entropy ${ }^{2}$ despite not directly accounting for the interactions formed by the hydrated solute with water.

THz fingerprint of bound water molecules. While the THz-fingerprints in the HB-stretching region probe cavity formation and associated entropic cost, we were lacking so far a direct $\mathrm{THz}$-fingerprint for the second step of our thermodynamic cycle, i.e. for water molecules H-Bonded to the alcohols $\mathrm{OH}$ group. To fill this gap, we move our attention from the HB-stretching region (100-300 $\left.\mathrm{cm}^{-1}\right)$ to the onset of the librational band of water $\left(300-600 \mathrm{~cm}^{-1}\right)$. In this frequency region we probe characteristic soft librations at lower frequencies $\left(<400 \mathrm{~cm}^{-1}\right)$, and hard librations at higher frequencies (400-600 $\left.\mathrm{cm}^{-1}\right)$, indicative of less or more hindered motions of the water molecules, respectively. In fig.3A, we report the difference $\mathrm{THz}$ spectrum of hydrated $\mathrm{tBuOH}$ with respect to bulk water for different temperatures. We systematically observe an absorption decrease below $400 \mathrm{~cm}^{-1}$, followed by an increase in the $400-600 \mathrm{~cm}^{-1}$ region. Similar trends are obtained for all the alcohols at various concentrations and temperatures in an extended frequency range compared to earlier measurements $^{31}$ (see SI and figure S1 for details). Fig.3B displays the theoretical $\mathrm{THz}$ spectrum calculated from 
ab initio MD simulations by considering only the contribution of bound water molecules around a single tBuOH solute (including both the self and cross correlation terms, see SI and ref. ${ }^{27}$ ) for comparison. Based on the

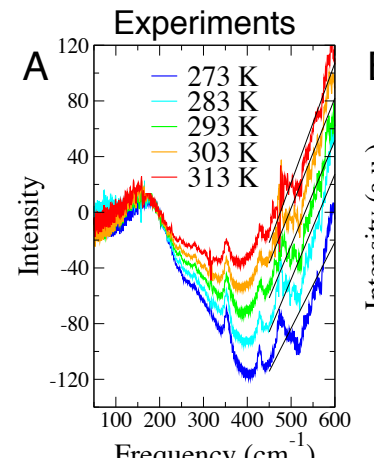

Frequency $\left(\mathrm{cm}^{-1}\right)$

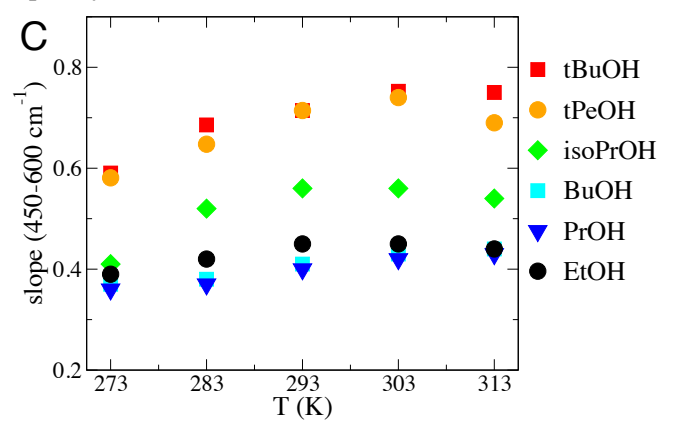

FIG. 3. THz signature of the bound water population. (A) Experimental hydration-shell resolved THz spectra of hydrated $t B u O H$ at various temperatures, showing a characteristic intensity increase in the 450-600 $\mathrm{cm}^{-1}$ region. The black solid lines are linear fits of the $\mathrm{THz}$ intensity in this region, from which the slope plotted in panel $C$ is obtained. (B) Theoretical THz spectrum calculated from DFT-MD (same as in ref. ${ }^{27}$ ) considering only the contribution (both self and cross correlation terms) of bound water molecules hydrating the polar $\mathrm{OH}$ group of $\mathrm{tBuOH}$. (C) Slope of the THz-intensity in the $450-600 \mathrm{~cm}^{-1}$ region for various experimentally investigated alcohols as a function of temperature.

theory-experiments comparison, we conclude that the absorption increase in the $450-600 \mathrm{~cm}^{-1}$ region is the direct spectroscopic signature of bound water molecules. These water molecules are indeed found from classical MD to have more constrained orientational motions as compared to the other water molecules in the hydration layer or in the bulk, which is consistent with a blue shift in the librational mode. In particular, we computed orientational lifetimes of 4.4 ps for bound waters, 2.9 ps for the remaining water molecules in the hydration layer, and $1.9 \mathrm{ps}$ for bulk water. This trend is well rationalized by the jump model of water reorientation: ${ }^{39}$ Water orientational dynamics is expected to slow down in the hydration layer ${ }^{40}$ due to an entropic excluded volume effect. ${ }^{41}$ Moreover, an additional enthalpic effect contributes to the slow down for bound water molecules as they form a $\mathrm{HB}$ with the $\mathrm{OH}$-group, further increasing the activation barrier for a jump. This is consistent with a population increase in the hard-librations and a loss in population of soft-librations, as observed experimentally. We want to mention that a similar absorption increase in the $\mathrm{THz}$ spectra at frequencies $>400 \mathrm{~cm}^{-1}$ has been previously reported for hydration water molecules as a result of strong interactions with ions or with polar groups of biomolecules. ${ }^{42-44}$ In order to quantify this effect, we take the slope of the absorption increase in the $450-600 \mathrm{~cm}^{-1}$ region and we compare it for different alcohols in Fig.3C. Remarkably, a similar slope is measured for EtOH, $\mathrm{PrOH}$ and $\mathrm{BuOH}$, which are all primary alcohols (i.e. the $\mathrm{C}$ atom carrying the $\mathrm{OH}$ group is bonded to one $\mathrm{C}$ atom only). The slope is independent on the number of apolar $\mathrm{CH}_{2}$ and $\mathrm{CH}_{3}$ groups contained in the alcohol under investigation. This confirms our assignment of the band to the bound water population at the alcohol OH-group. Accordingly, the slope changes as soon as the environment of the $\mathrm{OH}$ groups is altered, i.e. when going from primary to secondary (isoPrOH, for which the $\mathrm{C}$ atom carrying the $\mathrm{OH}$ group is bonded to two $\mathrm{C}$ atoms) and tertiary ( $\mathrm{tBuOH}, \mathrm{tPeOH})$ alcohols. This is attributed to increased steric hindrance for the water molecules in the surrounding of the $\mathrm{OH}$ group, as confirmed by classical MD simulations that predict a slower orientational dynamics for bound waters around tBuOH as compared to $\mathrm{BuOH}$ (4.4 vs 3.8 ps). This result is expected due to the increase in volume excluded effect around the $\mathrm{OH}$-group of a tertiary vs primary alcohol. ${ }^{39,41}$ As a final confirmation of our assignment, we note that the two tertiary alcohols have a different number of apolar groups, but the same slope.

In conclusion, alcohol hydration is well described by a two-step thermodynamic cycle, where (1) a cavity is formed in the liquid, and (2) a solute is inserted at the center of the cavity, switching on attractive solute-water interactions. The free energy cost of cavity formation (i.e. the hydrophobic cost) can be quantified experimentally via $\mathrm{THz}$-calorimetry by a characteristic HB-wrap mode at $164 \mathrm{~cm}^{-1}$, in the HB-stretching region. The free energy gain from step (2) is dominated by the H-Bonds formed between the alcohol $\mathrm{OH}$ group and bound water molecules. The latter provide a $\mathrm{THz}$ fingerprint in the $450-600 \mathrm{~cm}^{-1}$ librational region. $\mathrm{THz}$ spectroscopy hence allows directly probing independently both hydrophobic and hydrophilic contributions to hydration free energies, as summarized in Fig.4. By using $\mathrm{THz}$-calorimetry to relate spectroscopy to thermodynamics, we have shown that small alcohol hydration is a balance between entropic (hydrophobic) cost of cavity formation and enthalpic (hydrophilic) gain due to H-Bonds between the alcohol and bound waters. The entropic cost is almost independent on attractive solute-water interactions. Such behavior was previously predicted from theory for alkanes, ${ }^{45}$ which do not strongly interact with water. We show here that the same conclusion holds true for solutes such as alcohols 

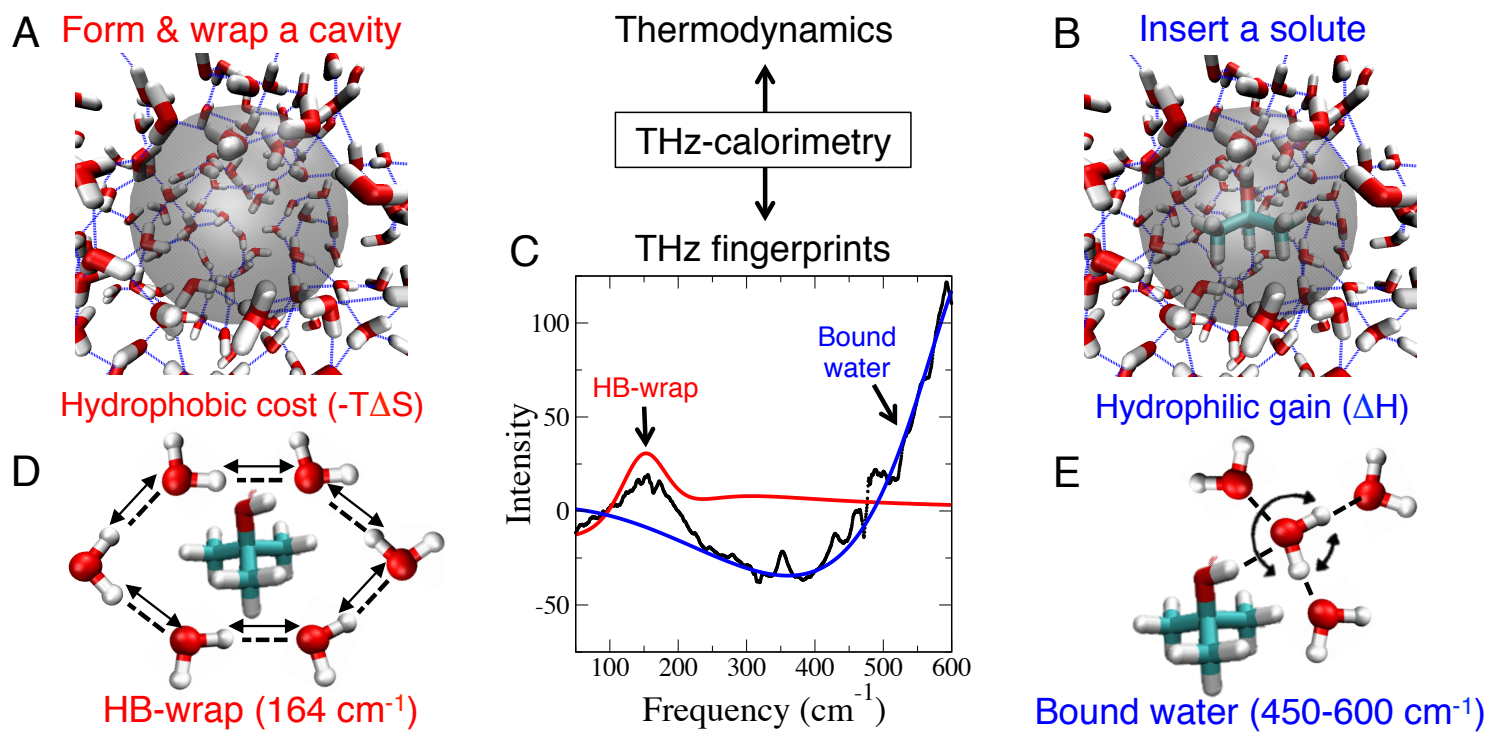

FIG. 4. Small alcohol hydration free energy is the sum of (A) an entropic cost of cavity formation, involving formation of a $H B$-wrap over the whole hydration layer, and $(B)$ an enthalpic gain due to the attractive interactions formed by the alcohol OH group with bound water molecules. (C) THz-calorimetry quantifies both terms from THz translational (due to HB-stretching, red) and librational (blue) fingerprints of the hydration layer. In the example, the THz spectrum at room $T$ is decomposed into the two terms for $\mathrm{tBuOH}$. (D) The $164 \mathrm{~cm}^{-1}$ THz fingerprint in the HB-stretching region probes the HB-wrap, while (E) the librational band probes bound water molecules.

possessing a polar group that can H-Bond water. This is possible because alcohol-water interactions do not require significant restructuring of the water network in the hydration layer (i.e. of the HB-wrap) with respect to the empty cavity limit. We propose that solutes with the described properties can be classified as "wrappable", since alcohol-water interactions are well commensurate to the HB-wrap formed in the hydration layer. Our framework anticipates an effect of the topological and morphological aspects of the solute (being it a small molecule or an extended surface) on its hydration free energy and solubility. Such effects were observed in previous theoretical studies ${ }^{16,26,46,47}$ of biological interfaces, and our approach offers a path for an experimental confirmation of these important findings.

In future studies, the $\mathrm{THz}$ fingerprints of wrap and bound hydration water populations can be used to rationalize and quantify local thermodynamic contributions to more complex biomolecules hydration. It will allow to experimentally dissect the role that hydrophobic and hydrophilic interactions play in many biological processes, such as biomolecular recognition, as well as in non equilibrium processes, and thereby allow to tune these properties separately for reaction steering.

\section{SUPPLEMENTARY MATERIAL}

See the supplementary material for experimental and computational details, as wall as for additional plots of the recorder $\mathrm{THz}$ spectra and the results of the $\mathrm{THz}-$ calorimetry analysis.

\section{ACKNOWLEDGMENTS}

We acknowledge financial support by European Research Council (ERC) Advanced Grant 695437 THz-Calorimetry. This study is funded by the Deutsche Forschungsgemeinschaft (DFG,German Research Foundation) under Germany's Excellence Strategy-EXC2033-390677874-RESOLV. We thank the Mercator Research Center Ruhr (MERCUR) for funding. This project has received funding from the European Union's Horizon 2020 programme (FP-RESOMUS - MSCA 801459). This work is supported by the "Center for Solvation Science ZEMOS" funded by the German Federal Ministry of Education and Research BMBF and by the Ministry of Culture and Research of Nord RhineWestphalia MKW NRW 


\section{AUTHOR DECLARATIONS}

\section{Conflict of interest}

The authors have no conflicts to disclose.

\section{DATA AVAILABILITY STATEMENT}

The data that support the findings of this study are available from the corresponding authors upon reasonable request.

${ }^{1}$ D. M. Huang and D. Chandler, "Temperature and length scale dependence of hydrophobic effects and their possible implications for protein folding," Proc. Natl. Acad. Sci. 97, 8324-8327 (2000). ${ }^{2}$ D. Chandler, "Interfaces and the driving force of hydrophobic assembly." Nature 437, 640-647 (2005).

${ }^{3}$ M. Liu, A. K. Das, J. Lincoff, S. Sasmal, S. Y. Cheng, R. M. Vernon, J. D. Forman-Kay, and T. Head-Gordon, "Configurational entropy of folded proteins and its importance for intrinsically disordered proteins," Int. J. Mol. Sci. 22, 3420 (2021).

${ }^{4} \mathrm{~J}$. Wereszczynski and J. A. McCammon, "Statistical mechanics and molecular dynamics in evaluating thermodynamic properties of biomolecular recognition," Q. Rev. Biophys. 45, 1-25 (2012).

${ }^{5}$ J. M. Fox, M. Zhao, M. J. Fink, K. Kang, and G. M. Whitesides, "The molecular origin of enthalpy/entropy compensation in biomolecular recognition," Annu. Rev. Biophys. 47, 223-250 (2018).

${ }^{6} \mathrm{M}$. Maurer and C. Oostenbrink, "Water in protein hydration and ligand recognition," J. Mol. Recognit. 32, e2810 (2019).

${ }^{7}$ A. Majumdar, P. Dogra, S. Maity, and S. Mukhopadhyay, "Liquid-liquid phase separation is driven by large-scale conformational unwinding and fluctuations of intrinsically disordered protein molecules," J. Phys. Chem. Lett. 10, 3929-3936 (2019).

${ }^{8}$ J. Ahlers, E. M. Adams, V. Bader, S. Pezzotti, K. F. Winklhofer, J. Tatzelt, and M. Havenith, "The key role of solvent in condensation: mapping water in liquid-liquid phase-separated fus," Biophys. J. 120, 1266-1275 (2021).

${ }^{9}$ A. Ianiro, H. Wu, M. M. van Rijt, M. P. Vena, A. D. Keizer, A. C. C. Esteves, R. Tuinier, H. Friedrich, N. A. Sommerdijk, and J. P. Patterson, "Liquid-liquid phase separation during amphiphilic self-assembly," Nat. chem. 11, 320-328 (2019).

${ }^{10}$ W.-L. Li and T. Head-Gordon, "Catalytic principles from natural enzymes and translational design strategies for synthetic catalysts," ACS central science 7, 72-80 (2020).

${ }^{11}$ F. Sebastiani, T. A. Bender, S. Pezzotti, W.-L. Li, G. Schwaab, R. G. Bergman, K. N. Raymond, F. D. Toste, T. Head-Gordon, and M. Havenith, "An isolated water droplet in the aqueous solution of a supramolecular tetrahedral cage," Proc. Natl. Acad. Sci. 117, 32954-32961 (2020).

${ }^{12}$ C. J. Hastings, M. D. Pluth, R. G. Bergman, and K. N. Raymond, "Enzymelike catalysis of the nazarov cyclization by supramolecular encapsulation," J.Am. Chem. Soc. 132, 69386940 (2010).

${ }^{13}$ G. Marcandalli, A. Goyal, and M. T. M. Koper, "Electrolyte effects on the faradaic efficiency of co2 reduction to co on a gold electrode," ACS Catal. 11, 4936-4945 (2021).

${ }^{14}$ A. Serva, M. Salanne, M. Havenith, and S. Pezzotti, "Size dependence of hydrophobic hydration at electrified gold/water interfaces," Proc. Natl. Acad. Sci. 118, e2023867118 (2021).

${ }^{15}$ P. Clabaut, B. Schweitzer, A. W. Götz, C. Michel, and S. N. Steinmann, "Solvation free energies and adsorption energies at the metal/water interface from hybrid quantummechanical/molecular mechanics simulations," J. Chem. Theory Comput. 16, 6539-6549 (2020).

${ }^{16}$ J. Monroe, M. Barry, A. DeStefano, P. A. Gokturk, S. Jiao, D. Robinson-Brown, T. Webber, E. J. Crumlin, S. Han, and
M. S. Shell, "Water structure and properties at hydrophilic and hydrophobic surfaces," Annu. Rev. Chem. Biomol. 11, 523-557 (2020).

${ }^{17}$ A. J. Patel, P. Varilly, and D. Chandler, "Fluctuations of water near extended hydrophobic and hydrophilic surfaces," J. Phys. Chem. B 114, 1632-1637 (2010).

${ }^{18}$ N. B. Rego, E. Xi, and A. J. Patel, "Identifying hydrophobic protein patches to inform protein interaction interfaces," Proc. Natl. Acad. Sci. 118, e2018234118 (2021).

${ }^{19}$ E. M. Adams, S. Pezzotti, J. Ahlers, M. Rüttermann, M. Levin, A. Goldenzweig, Y. Peleg, S. J. Fleishman, I. Sagi, and M. Havenith, "Local mutations can serve as a game changer for global protein solvent interaction," JACS Au 1, 1076-1085 (2021).

${ }^{20}$ A. Serva, , M. Havenith, and S. Pezzotti, "The role of hydrophobic hydration in the free energy of chemical reactions at the gold/water interface: size and position effects," J. Chem. Phys. 155, 204706 (2021).

${ }^{21}$ E. Duboué-Dijon and D. Laage, "Characterization of the local structure in liquid water by various order parameters," J. Phys. Chem. B 119, 8406-8418 (2015).

${ }^{22} \mathrm{~N}$. Galamba, "Free energy convergence in short-and long-length hydrophobic hydration," J. Mol. Liq. 339, 116699 (2021).

${ }^{23}$ N. Giovambattista, P. J. Rossky, and P. G. Debenedetti, "Effect of temperature on the structure and phase behavior of water confined by hydrophobic, hydrophilic, and heterogeneous surfaces," J. Phys. Chem. B 113, 13723-13734 (2009).

${ }^{24}$ N. Giovambattista, P. G. Debenedetti, and P. J. Rossky, "Hydration behavior under confinement by nanoscale surfaces with patterned hydrophobicity and hydrophilicity," J. Phys. Chem. C 111, 1323-1332 (2007).

${ }^{25}$ J. G. Davis, K. P. Gierszal, P. Wang, and D. Ben-Amotz, "Water structural transformation at molecular hydrophobic interfaces," Nature 491, 582-585 (2012).

${ }^{26}$ S. N. Jamadagni, R. Godawat, and S. Garde, "Hydrophobicity of proteins and interfaces: Insights from density fluctuations," Annu. Rev. Chem. Biomol. Eng. 2, 147-171 (2011).

${ }^{27}$ V. Conti Nibali, S. Pezzotti, F. Sebastiani, D. R. Galimberti, G. Schwaab, M. Heyden, M.-P. Gaigeot, and M. Havenith, "Wrapping up hydrophobic hydration: Locality matters," J. Phys. Chem. Letters 11, 4809-4816 (2020).

${ }^{28}$ N. Abidi, K. R. G. Lim, Z. W. Seh, and S. N. Steinmann, "Atomistic modeling of electrocatalysis: Are we there yet?" WIREs Comput. Mol. Sci. 11, e1499 (2021).

${ }^{29}$ S. E. Sanders and P. B. Petersen, "Heterodyne-detected sum frequency generation of water at surfaces with varying hydrophobicity," J. Chem. Phys. 150, 204708 (2019).

${ }^{30}$ Y. Yang, F. C. Lightstone, and S. E. Wong, "Approaches to efficiently estimate solvation and explicit water energetics in ligand binding: the use of watermap," Expert Opin Drug Discov 8, 277-287 (2013).

${ }^{31}$ F. Bohm, G. Schwaab, and M. Havenith, "Mapping hydration water around alcohol chains by thz calorimetry," Angew. Chem. Int. Ed. 56, 9981-9985 (2017).

${ }^{32} \mathrm{P}$. V. David Chandler, "Lectures on molecular- and nano-scale fluctuations in water," Proceedings of the International School of Physics "Enrico Fermi" 176, 75-111 (2012).

${ }^{33}$ K. Lum, D. Chandler, and J. D. Weeks, "Hydrophobicity at small and large length scales," J. Phys. Chem. B 103, 4570-4577 (1999).

${ }^{34}$ S. Funke, F. Sebastiani, G. Schwaab, and M. Havenith, "Spectroscopic fingerprints in the low frequency spectrum of ice (ih), clathrate hydrates, supercooled water, and hydrophobic hydration reveal similarities in the hydrogen bond network motifs," J. Chem. Phys. 150, 224505 (2019).

${ }^{35}$ D. Ben-Amotz, "Hydration-shell vibrational spectroscopy," J. Am. Chem. Soc. 141, 10569-10580 (2019).

${ }^{36}$ A. J. Bredt and D. Ben-Amotz, "Influence of crowding on hydrophobic hydration-shell structure," Phys. Chem. Chem. Phys. 22, 11724-11730 (2020). 
${ }^{37}$ S. Pezzotti, A. Serva, F. Sebastiani, F. S. Brigiano, D. R. Galimberti, L. Potier, S. Alfarano, G. Schwaab, M. Havenith, and M.P. Gaigeot, "Molecular fingerprints of hydrophobicity at aqueous interfaces from theory and vibrational spectroscopies," J. Phys. Chem. Lett. 12, 3827-3836 (2021).

${ }^{38}$ R. A. X. Persson, V. Pattni, A. Singh, S. M. Kast, and M. Heyden, "Signatures of solvation thermodynamics in spectra of intermolecular vibrations," J. Chem. Theory Comput. 13, 4467-4481 (2017).

${ }^{39}$ D. Laage and J. T. Hynes, "A molecular jump mechanism of water reorientation," Science 311, 832-835 (2006).

${ }^{40}$ A. A. Bakulin, M. S. Pshenichnikov, H. J. Bakker, and C. Petersen, "Hydrophobic molecules slow down the hydrogen-bond dynamics of water," J. Phys. Chem. A 115, 1821-1829 (2011).

${ }^{41}$ D. Laage, G. Stirnemann, and J. T. Hynes, "Why water reorientation slows without iceberg formation around hydrophobic solutes," J. Phys. Chem. B 113, 2428-2435 (2009).

${ }^{42}$ P. Schienbein, G. Schwaab, H. Forbert, M. Havenith, and D. Marx, "Correlations in the solute-solvent dynamics reach beyond the first hydration shell of ions," J. Phys. Chem. Lett. 8,
2373-2380 (2017).

${ }^{43}$ F. Sebastiani, C. Y. Ma, S. Funke, A. Bäumer, D. Decka, C. Hoberg, A. Esser, H. Forbert, G. Schwaab, D. Marx, and M. Havenith, "Probing local electrostatics of glycine in aqueous solution by thz spectroscopy," Angew. Chem. Int. Ed. 60, 37683772 (2021).

${ }^{44}$ C. Y. Ma, S. Pezzotti, G. Schwaab, M. Gebala, D. Herschlag, and M. Havenith, "Cation enrichment in the ion atmosphere is promoted by local hydration of dna," Phys. Chem. Chem. Phys. 23, 23203-23213 (2021).

${ }^{45}$ E. Gallicchio, M. Kubo, and R. M. Levy, "Enthalpy- entropy and cavity decomposition of alkane hydration free energies: $\mathrm{Nu}-$ merical results and implications for theories of hydrophobic solvation," J. Phys. Chem. B 104, 6271-6285 (2000).

${ }^{46}$ J. I. Monroe and M. S. Shell, "Computational discovery of chemically patterned surfaces that effect unique hydration water dynamics," Proc. Natl. Acad. Sci. 115, 8093-8098 (2018).

${ }^{47}$ E. Xi, V. Venkateshwaran, L. Li, N. Rego, A. Patel, and S. Garde, "Hydrophobicity of proteins and nanostructured solutes is governed by topographical and chemical context," Proc. Natl. Acad. Sci. 114, 201700092 (2017). 